\title{
A State Observer Design for Simultaneous Estimation of Charge State and Crossover in Self-Discharging Disproportionation Redox Flow Batteries
}

\author{
Pedro Ascencio*, Kirk Smith, David Howey, and Charles W. Monroe
}

\begin{abstract}
This paper presents an augmented state observer design for the simultaneous estimation of charge state and crossover flux in disproportionation redox flow batteries, which exhibits exponential estimation error convergence to a bounded residual set. The crossover flux of vanadium through the porous separator is considered as an unknown function of the battery states, model-approximated as the output of a persistently excited linear system. This parametric model and the simple isothermal lumped parameter model of the battery are combined to form an augmented space state representation suitable for the observer design, which is carried out via Lyapunov stability theory including the error-uncertainty involved in the approximation of the crossover flux. The observer gain is calculated by solving a polytopic linear matrix inequality problem via convex optimization. The performance of this design is evaluated with a laboratory flow battery prototype undergoing self-discharge.
\end{abstract}

\section{INTRODUCTION}

Many multi-physics models have been developed to improve the design and performance of redox flow batteries (RFBs), in particular for aqueous all-vanadium chemistries [1], [2], [3]. More elaborate models include distributed parameter descriptions, for instance considering mass transport by diffusion, convection, and migration, among other dynamics [4], [5], [6], [7]. Alternatively, computationally tractable lumped-parameter models assume pseudo-steady transport of solute across the separator, expressing a proportionality between species fluxes and their concentration differences [8], [9], [10], [11].

Basic approaches to RFB modelling usually assume perfect reservoir balancing and membrane selectivity, but RFB efficiency and lifetime are both affected by the persistent crossover of active species through the separator [12], [13], [14], [15], [16], [6]. Crossover is usually modelled as Fickian diffusion [17], [18], [19], [20], avoiding more complex transport behaviour within the membrane [21], [22]. This sort of phenomenological simplification, alongside the assumption that model parameters remain constant during operation ${ }^{1}$ can lead to erroneous performance predictions.

To enable more accurate prediction of RFB behaviour and to gain more detailed knowledge about physico-chemical

Pedro Ascencio (pedro.ascencio@eng.ox.ac.uk), Kirk Smith (kirk.smith@eng.ox.ac.uk), Prof. David Howey (david.howey@eng.ox.ac.uk) and Prof. Charles W. Monroe (charles.monroe@eng.ox.ac.uk) are with the Department of Engineering Science, University of Oxford, Oxford OX1 3PJ, United Kingdom. ${ }^{*}$ Corresponding author.

${ }^{1}$ In general, parameters of these models are assumed known by means of standard experiments or model fits (e.g. [23]). changes, observer-based approaches for lumped parameter models of RFBs have been developed. In some cases such models can provide simultaneous and continuous estimation of the main battery states and parameters [24], [25], [26], [27], [28]. Generally, these approaches use electrical equivalent-circuit models (ECMs) to emulate the RFB's electrical behaviour, and perform state/parameter estimation with an extended Kalman filter (EKF) [24], [29], [25], [28]. ECMs do not explicitly describe the crossover flux that causes self-discharge or irreversible degradation; the consequences of crossover are deduced or detected from the transient observer-predicted variables and parameters.

This paper addresses the simultaneous estimation of battery states and crossover flux for a novel type of RFB based on disproportionation chemistry - a so-called DRFB [30], [31]. The battery is described with a simple isothermal lumped-parameter model, which considers the state of charge in one half-cell (including the associated reservoir) and in one chamber of the reactor, alongside the crossover flux out of the half-cell through the reactor's membrane separator. This paper presents an alternative approach to previous work by the authors [32] which included a general parametric model for the crossover flux with no particular assumptions about a specific transport mechanism through the membrane. The present model instead considers time varying parameters generated by a persistently excited linear system, whose states are included as additional states in the original nonlinear DRFB model, and whose space-state representation is suitable for observer design based on the standard Lyapunov second method of stability. The gain of the observer is numerically computed solving the resulting linear matrix inequality (LMI) problem via convex optimization, using a polytopic approach.

\section{A. Notation}

The space of all continuous functions on the domain $\Omega$ into $\mathbb{R}$ is denoted $\mathcal{C}(\Omega ; \mathbb{R})$. The set of symmetric positive definite matrices of dimension $n \times n$ is denoted $\mathbb{S}_{++}^{n} ; \mathbf{0}_{n \times m}$ is the null matrix of dimension $n \times m$ and $\boldsymbol{I}_{n}$ is the identity matrix of dimension $n \times n$. Also, $\mathbb{R}^{+} \equiv[0,+\infty)$, $\mathbb{R}^{m+}=\mathbb{R}^{+} \times \ldots \times \mathbb{R}^{+}$in $m \in \mathbb{N}$ times, $\dot{v}(t) \triangleq \frac{d v}{d t}(t)$, $\|x\| \triangleq \sqrt{x^{\top} x}$ with $x \in \mathbb{R}^{n}$ being a real vector of dimension $n$, and $\|E\| \triangleq \sqrt{\lambda_{\max }\left(E^{\top} E\right)}$ with $E$ being a real matrix, where $\lambda_{\max }$ denotes the largest eigenvalue; $\lambda_{\min }$ stands for the smallest eigenvalue. 


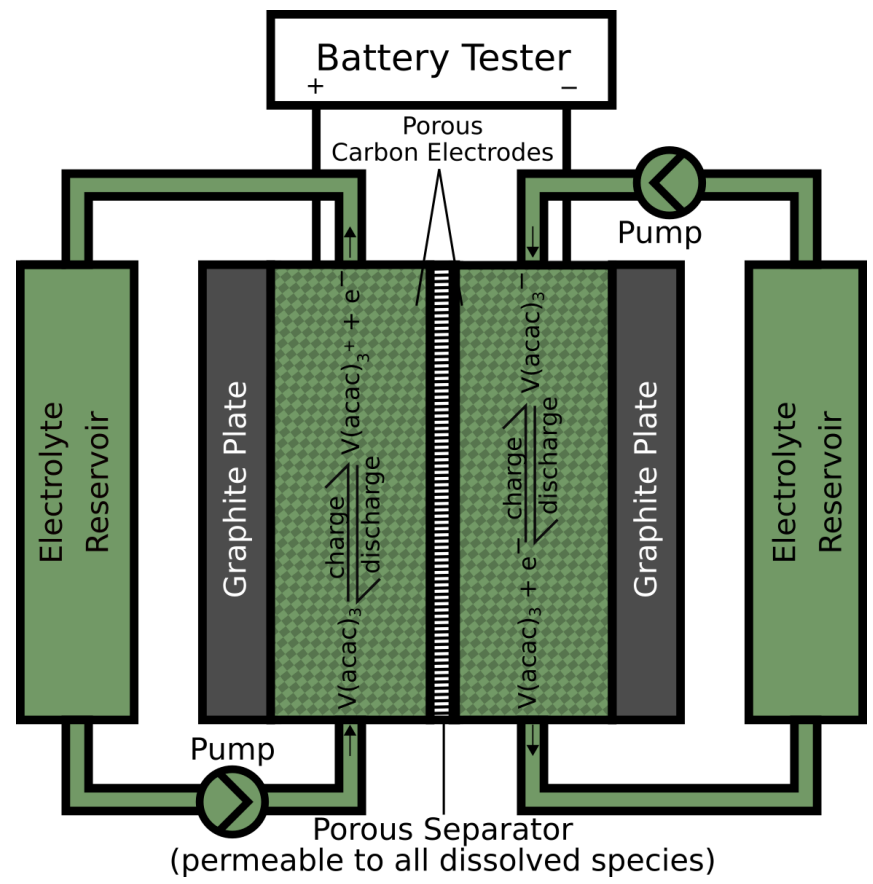

Fig. 1: Schematic of a DRFB using vanadium acetylacetonate

\section{Modeling DRFBS}

The DRFB we analyse contains two separated reservoirs storing identical liquid electrolytic solutions in the fully discharged state. The discharged cell contains a single metal electroactive species, vanadium acetylacetonate ( $\mathrm{V}(\mathrm{acac}) 3)$, which can be both oxidized and reduced (see Figure 1 . This species has been shown to allow a disproportionation electrochemistry, i.e. the battery's charging process causes $\mathrm{V}(\mathrm{acac}) 3$ to oxidize on one side of the battery, while it reduces on the other. A key feature of this DRFB is the use of a nonaqueous solvent, which allows the high redox potential associated with $\mathrm{V}$ (acac) 3 disproportionation. The DRFB configuration may make costly selective separator membranes and periodic active-liquid regeneration unnecessary [31], if suitable modes of control can be developed to maintain relatively high energy efficiency during cycling.

In accordance with [30], cyclic voltammetry experiments show two main redox couples associated with $\mathrm{V}(\mathrm{acac}) 3$ :

- Negative Chamber

$$
\mathrm{V}(\mathrm{III})(\text { acac }) 3+\mathrm{e}^{-} \underset{\text { discharge }}{\stackrel{\text { charge }}{\rightleftharpoons}}[\mathrm{V}(\mathrm{II})(\mathrm{acac}) 3]^{-} \text {, }
$$

- Positive Chamber

$$
\mathrm{V}(\mathrm{III})(\mathrm{acac}) 3 \underset{\text { discharge }}{\stackrel{\text { charge }}{\rightleftharpoons}}[\mathrm{V}(\mathrm{IV})(\mathrm{acac}) 3]^{+}+\mathrm{e}^{-} \text {. }
$$

Together these establish an equilibrium cell potential of $E^{0}=2.18$ [V] [30], [22]. The reversible nature of the reactions makes this type of battery tolerant to crossover electrolyte flux. Crossover causes comproportionation of $\mathrm{V}(\mathrm{II})$ and $\mathrm{V}(\mathrm{IV})$ to form $\mathrm{V}(\mathrm{III})(\mathrm{acac}) 3$ [22], leading to self-discharge, but does not permanently degrade the battery capacity. DRFBs thus enable porous separators to be used, opening up a value tradeoff between the reactor capital cost and its coulombic efficiency [14], [30], [22], [33], [31].

\section{A. Isothermal Lumped Parameter Model}

In this section, for clarity of explanation, the model formulated by the authors in [32] is described.

Due to the symmetry of the disproportionation chemistry, under identical flows supplied by synchronised pumps, only one side of the battery needs to be analysed to model discharge. In isothermal operation, let $n$ and $n_{\text {cell }}$ be the amount of neutral $\mathrm{V}(\mathrm{acac}) 3$ species remaining in one reservoir and in the half-cell, respectively. The rate of change of $n$ is due to the current $I$ driven by the battery, and the crossover flux $Q_{x}$ through the separator, namely

$$
\frac{d n}{d t}(t)=\frac{I(t)}{\mathcal{F}}+Q_{x}(s(t))
$$

where $\mathcal{F}$ is the Faraday's constant and $Q_{x}$ is considered to be a function of possible $m \in \mathbb{N}$ variables/states $s$ of the battery, which could be states, inputs or outputs of the model, or even other measurements, independent of the model: $s: \mathbb{R}^{+} \rightarrow \Omega \subset \mathbb{R}^{m+}$. Thus, considering the halfcell reactor volume $V_{\text {cell }}$ to be negligible with respect to to the volume in the reservoir $V_{\text {res }}$ and $c(t)=n(t) / V_{\text {res }}$ being overall concentration of neutral species, the rate of change of the overall system state-of-charge, defined by $S O C(t)=\left(n_{0}-n(t)\right) / n_{0}=\frac{c_{0}-c(t)}{c_{0}}$, can be described by

$$
\frac{d S O C}{d t}(t)=\left(\frac{-1}{c_{0} V_{\mathrm{res}}}\right) Q_{x}(s(t))-\left(\frac{1}{c_{0} V_{\mathrm{res}} \mathcal{F}}\right) I(t),
$$

with $c_{0}=n_{0} / V_{\text {res }}$ as initial concentration. Accordingly, based on the principle of conservation of mass applied to one half-cell, assuming perfect mixing (similarly to a continuous stirred tank reactor model) and $c_{\text {cell }}(t)=n_{\text {cell }}(t) /\left(\epsilon V_{\text {cell }}\right)$ being the concentration of neutral species in the half-cell, the rate of change of the reactor state-of-charge, defined by $S O C_{\text {cell }}(t)=\frac{c_{0}-c_{\text {cell }}(t)}{c_{0}}$, can be expressed by

$$
\frac{d S O C_{\text {cell }}}{d t}(t)=\left(\frac{-1}{\epsilon c_{0} V_{\text {cell }}}\right) \frac{d n}{d t}(t)-\left(\frac{Q(t)}{\epsilon V_{\text {cell }}}\right) \Delta S O C(t),
$$

with $\frac{d\left(n_{\text {cell }}-n\right)}{d t}(t)=Q(t)\left(c(t)-c_{\text {cell }}(t)\right)$, where $Q$ stands for the volumetric flow rate in the reactor (considered measurable and equal in both chambers) and $\triangle S O C(t)=$ $S O C_{\text {cell }}(t)-S O C(t) ; \epsilon$ accouts for the known porosity of the carbon electrode. Therefore, from (2)-(3), the resulting space-state isothermal lumped parameter model for the DRFB is

$$
\begin{aligned}
& {\left[\begin{array}{c}
\frac{d S O C}{d t}(t) \\
\frac{d S O C_{\text {cell }}}{d t}(t)
\end{array}\right]=\underbrace{\left.\left(\begin{array}{cc}
0 & 0 \\
\frac{Q(t)}{\epsilon V_{\text {cell }}} & \left.-\frac{Q(t)}{\epsilon V_{\text {cell }}}\right)
\end{array}\right] \begin{array}{c}
S O C(t) \\
S O C_{\text {cell }}(t)
\end{array}\right]+}_{A(Q(t))}} \\
& \underbrace{\left[\begin{array}{c}
-\frac{1}{c_{0} V_{\text {res }}} \\
-\frac{1}{\epsilon c_{0} V_{\text {cell }}}
\end{array}\right]}_{E} Q_{x}(s(t))+\underbrace{\left[\begin{array}{c}
-\frac{1}{c_{0} V_{\text {res }} \mathcal{F}} \\
-\frac{1}{\epsilon c_{0} V_{\text {cell }} \mathcal{F}}
\end{array}\right]}_{B} I(t),
\end{aligned}
$$

\footnotetext{
${ }^{2}$ Positive current is considered a discharge process.
} 


$$
V_{\text {out }}(t)=\underbrace{E_{\text {cell }}^{0}+\frac{2 \mathcal{R} \mathcal{T}}{\mathcal{F}} \ln \left(\frac{S O C_{\text {cell }}(t)}{1-S O C_{\text {cell }}(t)}\right)+V_{R}(t)}_{\Gamma\left(S O C_{\text {cell }}(t), I(t)\right)},
$$

where $V_{\text {out }}$ denotes the output voltage of the battery based on the Nernst equation, $\mathcal{R}$ is the universal gas constant, $\mathcal{T}=$ $275{ }^{\circ} \mathrm{K}$ and $V_{R}=V_{R}\left(S O C_{\text {cell }}(t), I(t)\right)$ considers voltage drops due to overpotentials $\left(V_{R}\left(S O C_{\text {cell }}(t), 0\right)=0\right)$.

\section{B. Augmented Parametric Model for Crossover Flux}

The unknown crossover flux in the DRFB can be considered to be a continuous function of the battery variables. Thus, this dynamic can be modelled approximately as the output of a linear system excited by randomly occurring impulses [34]. A particular case of this approach consists in considering the unknown parameter $\theta$ as the output of a $l$-th pure integrator: $\frac{d^{l} \theta}{d t^{l}}(t)=0$ [35], [36], namely

$$
\begin{aligned}
Q_{x}(s(t)) & =\Psi(s(t)) \theta(t)+\varepsilon(t), \\
\frac{d \omega}{d t}(t) & =\Lambda(\lambda) \omega(t), \\
\theta(t) & =[1,0, \ldots, 0] \omega(t)=\mathbf{0}_{l}, \\
\Lambda & =\left(\begin{array}{ccccc}
0 & \lambda_{1} & 0 & \ldots & 0 \\
0 & 0 & \lambda_{2} & \ldots & \vdots \\
\vdots & \vdots & \vdots & \ddots & \vdots \\
0 & 0 & 0 & \ldots & \lambda_{l-1} \\
0 & 0 & \ldots & \ldots & 0
\end{array}\right)
\end{aligned}
$$

where $\Psi \in \mathcal{C}(\bar{\Omega} \supseteq \Omega ; \mathbb{R})$ is a continuous and invertible function, $\varepsilon \in \mathbb{R}^{m}$ is an unknown dynamic error in the linear parametric model, and $\theta$ is extended to $l$ states $\omega(t)=\left[\omega_{1}(t), \ldots, \omega_{l}(t)\right]^{\top}: \mathbb{R}^{+} \rightarrow \mathbb{R}^{l}$, the dynamic of which is characterised by the matrix $\Lambda \in \mathbb{R}^{l \times l}$ for some $\lambda=\left[\lambda_{1}, \ldots, \lambda_{i}, \ldots, \lambda_{l-1}\right]^{\top}$ with $\lambda_{i} \in \mathbb{R}^{+}$gains of each parameter state.

\section{General Approximate Model}

Let $z(t)=\left[S O C(t), S O C_{\text {cell }}(t)\right]^{\top}$ be the vector of battery states, $z: \mathbb{R}^{+} \rightarrow \mathcal{Z} \subset \mathbb{R}^{2+}$. For the augmented state vector $x(t)=\left[z^{\top}(t), \theta(t), \omega_{2}(t), \ldots, \omega_{l}(t)\right]^{\top} \in \mathbb{R}^{l+2}\left(\theta=\omega_{1}\right)$, using the model-approximation of the crossover function (6), the DRFB model (4)-(5) can be formulated as

$$
\begin{aligned}
& \dot{x}(t)=A_{e}(\Psi(s(t)), Q(t)) x(t)+B_{e} I(t)+E_{e} \varepsilon(t), \\
& y(t)=\Gamma^{-1}\left(V_{\text {out }}(t), I(t), V_{R}(t)\right)=\underbrace{\left[0,1,0, \mathbf{0}_{1 \times l-1}\right.}_{C_{e}}] x(t), \\
& A_{e}=\left(\begin{array}{ccc}
Q(t) A_{\text {cell }} & E \Psi(s(t)) & \mathbf{0}_{2 \times l-1} \\
\mathbf{0}_{l-1 \times 2} & \mathbf{0}_{l-1 \times 1} & M(\lambda) \\
\mathbf{0}_{1 \times 2} & 0 & \mathbf{0}_{1 \times l-1}
\end{array}\right) \in \mathbb{R}^{C_{e}} \\
& A_{\text {cell }}=\left(\begin{array}{cc}
0 & 0 \\
\frac{1}{\epsilon V_{\text {cell }}} & -\frac{1}{\epsilon V_{\text {cell }}}
\end{array}\right) \text {, }
\end{aligned}
$$

where $x(0)=x_{0}$ is the unknown state and parameter initial condition, $y$ is the measurable battery output, $E_{e}=\left[E ; \mathbf{0}_{l \times 1}\right]$, $B_{e}=\left[B ; \mathbf{0}_{l \times 1}\right]$ and $M=\operatorname{diag}(\lambda) \in \mathbb{R}^{l-1 \times l-1}$ is a diagonal non-singular matrix $3^{3}$ It is assumed that the augmented model (7) satisfies the following conditions:

Condition 1 The functional structure of the $V_{R}$ term in (5) is such that the non-linear mapping $\Gamma$ is globally invertibl ${ }^{4}$

Condition 2 The battery states $z$ are always bounded: $\sup _{t}\{\|z(t)\|\} \leq \gamma_{z}, \forall t \in \mathbb{R}^{+}$, for some $\gamma_{z} \in \mathbb{R}^{+}$. In addition, the crossover flux represents a slow degradation process such that in (7) there exist bounded parameters: $\sup _{t}\{\|\theta(t)\|\} \leq \gamma_{\theta}$ and $\sup _{t}\{\|\omega(t)\|\} \leq \gamma_{\omega}$ which lead to a bounded approximation error: $\sup _{t}\{\|\varepsilon(s(t))\|\} \leq \bar{\varepsilon}$ in (6), $\forall t \in \mathbb{R}^{+}$, for some $\gamma_{\theta} \in \mathbb{R}^{+}, \gamma_{\omega} \in \mathbb{R}^{+}$and $\bar{\varepsilon} \in \mathbb{R}^{+}$.

Condition 3 The function $\Psi$ in (6) is not singular and always bounded: $\sup _{t}\{\|\Psi(\hat{s}(t))\|\} \leq \gamma_{\Psi}, \forall t \in \mathbb{R}^{+}$, for $\hat{s}: \mathbb{R}^{+} \rightarrow \bar{\Omega} \subset \mathbb{R}^{m+} ; \bar{\Omega} \supseteq \Omega$, and for some $\gamma_{\Psi} \in \mathbb{R}^{+}$. In addition, this satisfies the Lipchitz condition: $\| \Psi(s(t))-$ $\Psi(\hat{s}(t))\left\|\leq \gamma_{\tilde{\Psi}}\right\| s(t)-\hat{s}(t) \|$, for some $\gamma_{\tilde{\Psi}} \in \mathbb{R}^{+}, \forall s:$ $\mathbb{R}^{+} \rightarrow \Omega$. The battery state-variables $s$ are such that $\|\tilde{s}(t)\|=\|s(t)-\hat{s}(t)\| \leq \gamma_{\tilde{s}}\|\tilde{z}(t)\|$ for some $\gamma_{\tilde{s}} \in \mathbb{R}^{+}$, with $\tilde{z}(t)=z(t)-\hat{z}(t), \hat{z}: \mathbb{R}^{+} \rightarrow \overline{\mathcal{Z}} \subset \mathbb{R}^{2^{+}}, \overline{\mathcal{Z}} \supseteq \mathcal{Z}, \forall t \in \mathbb{R}^{+}$.

\section{OBSERVER DESIGN}

For the approximate model (7), the observer design can be carried out using Lyapunov stability theory [37] based on high gain observer approaches [38], [39], [40], [41]. The use of the parametric model (6) and the derivation of the LMI problem aim to improve the tracking performance of the states with a lower observer gain [42], [43], [36], [44]. The objective is to achieve stability in the sense of exponential uniformly ultimately bounded (EUUB) convergence of the observer estimation error [38].

\section{A. State Observer}

The proposed high gain-type observer [45] for simultaneous estimation of battery state and parameters for the system (7) has the structure

$$
\begin{aligned}
\dot{\hat{x}}(t) & =A_{e}(\Psi(\hat{s}(t)), Q(t)) \hat{x}(t)+B_{e} I(t)+H_{t} \tilde{y}(t) \\
\hat{y}(t) & =C_{e} \hat{x}(t),
\end{aligned}
$$

where $\hat{x}=\left[\hat{z}^{\top}, \hat{\theta}, \hat{\omega}_{2}, \ldots \hat{\omega}_{l}\right]^{\top}$ and $\hat{y}$ are respectively the estimated augmented state vector (including parameters), and the estimated outputs, $\hat{x}(0)=\hat{x}_{0}$ are estimated initial conditions, $\hat{s}$ stands for the estimated states/variables of the battery chosen to model the crossover flux, $\tilde{y}(t)=y(t)-\hat{y}(t)$ denotes the output estimation error and $H_{t} \in \mathbb{R}^{l+2}$ is the time-varying observer linear feedback gain. The following arguments will be instrumental in the observer design procedure:

\footnotetext{
${ }^{3}$ In terms of the observer design, matrices $A_{e}, B_{e}, C_{e}$ and $E_{e}$ are considered known or part of the design. The inversion of $\Gamma$ is based on Condition 1 for small errors in the voltage measurements. The symbols "," and "," in the matrix-vector notation denotes a (Matlab type) row and column separator, respectively.

${ }^{4}$ In particular, under open-circuit conditions since $V_{R}\left(S O C_{\text {cell }}(t), 0\right)=$ 0 , for $S O C_{\text {cell }}(t) \neq 1, \forall t \in \mathbb{R}^{+}$(monotonic property of the logarithm function).
} 
Definition 1 By virtue of Condition 3 the matrix transformation defined by

$$
\hat{T}(t)=T(\Psi(\hat{s}(t)))=\left(\begin{array}{cc}
\boldsymbol{I}_{2} & \mathbf{0}_{2 \times l} \\
\mathbf{0}_{l \times 2} & (1 / \varrho) \Psi(\hat{s}(t)) \boldsymbol{I}_{l}
\end{array}\right),
$$

for some $\varrho \in(0,+\infty)$, is invertible and it verifies:

$$
\begin{aligned}
\hat{T}(t) A_{e}(\Psi(\hat{s}(t)), Q(t)) \hat{T}^{-1}(t)=\mathcal{A}(Q(t)), & \\
= & \left(\begin{array}{ccc}
Q(t) A_{\text {cell }} & \varrho E & \mathbf{0}_{2 \times l-1} \\
\mathbf{0}_{l-1 \times 2} & \mathbf{0}_{l-1 \times 1} & M(\lambda) \\
\mathbf{0}_{1 \times 2} & 0 & \mathbf{0}_{1 \times l-1}
\end{array}\right),
\end{aligned}
$$

for every $\hat{s}: \mathbb{R}^{+} \rightarrow \bar{\Omega}, \forall t \in \mathbb{R}^{+}$, so that $\mathcal{A}$ does not depend on $\Psi$.

Condition 4 Based on Condition 3 the invertible transformation (9) is always bounded in the following sense:

$$
\begin{aligned}
& \tau_{m}=\inf _{t \in \mathbb{R}^{+}}\left\{1, \frac{|\Psi(\hat{s}(t))|}{\varrho}\right\} \leq\|\hat{T}(t)\| \leq \sup _{t \in \mathbb{R}^{+}}\left\{1, \frac{|\Psi(\hat{s}(t))|}{\varrho}\right\}=\tau_{M}, \\
& \left\|\dot{\hat{T}}(t) \hat{T}^{-1}(t)\right\| \leq \gamma_{T}=\sup _{t \in \mathbb{R}^{+}}\left\{1,\left|\frac{d \Psi(\hat{s}(t))}{d t} \Psi^{-1}(\hat{s}(t))\right|\right\},
\end{aligned}
$$

for every $\hat{s}: \mathbb{R}^{+} \rightarrow \bar{\Omega}$, for some $\gamma_{T}>0, \tau_{m}>0$ and $\tau_{M}>0$, $\forall t \in \mathbb{R}^{+}$.

Condition 5 The volumetric flow rate $Q$ is a bounded measurable variable with $Q_{m}=\inf _{t}\{Q(t)\}>0$ and $Q_{M}=$ $\sup _{t}\{Q(t)\}$, so that its domain of operation $\mathcal{Q}=\left[Q_{m}, Q_{M}\right]$ is a compact set known in advance. Thus, the time-varying matrix $\mathcal{A}$ in 10 can always be embedded in a polytope of matrices:

$$
\mathcal{A}(Q(t)) \in \mathcal{P}=\mathbf{C o}\left\{A\left(Q_{m}\right), A\left(Q_{M}\right)\right\},
$$

$\forall t \in \mathbb{R}^{+}$, where Co denotes the convex hull (minimal convex polytope) [46], [47].

\section{B. Observer Estimation Error}

The dynamical error between the model (7) and proposed observer (8) can be expressed as the following linear perturbed dynamic model [48]:

$$
\begin{aligned}
& \dot{\tilde{x}}(t)=\bar{A}_{e}(t) \tilde{x}(t)+\eta(t), \\
& \eta(t)=\tilde{A}_{e}(t) x(t)+E_{e} \varepsilon(t), \\
& \tilde{y}(t)=C_{e} \tilde{x}(t),
\end{aligned}
$$

where $\tilde{x}(t)=x(t)-\hat{x}(t)$ and $\tilde{y}(t)=y(t)-\hat{y}(t)$ are estimation errors for states/parameters and outputs, respectively, with $\tilde{x}=0$ being the only stable equilibrium point of the nominal system and $\tilde{x}(0)=\tilde{x}_{0}$ are initial error conditions; $\bar{A}_{e}(t)=\hat{A}_{e}(t)-H_{t} C_{e}, \tilde{A}_{e}(t)=A_{e}(t)-\hat{A}_{e}(t)$, with $\hat{A}_{e}(t)=$ $A_{e}(\Psi(\hat{s}(t)), Q(t))$ and $A_{e}(t)=A_{e}(\Psi(s(t)), Q(t))$. Thus, by meas of the invertible transformation (9), given in Definition 1. the observer estimation error (11) can be written as:

$$
\begin{aligned}
& e(t)=\hat{T}(t) \tilde{x}(t), \\
& \dot{e}(t)=\left(\hat{T}(t) \bar{A}_{e} \hat{T}^{-1}(t)\right) e(t)+\left(\dot{\hat{T}}(t) \hat{T}^{-1}(t)\right) e(t)+\hat{T}(t) \eta(t), \\
& =\underbrace{\left(\mathcal{A}(Q(t))-\hat{T}(t) H_{t} C_{e}\right)}_{\overline{\mathcal{A}}(t)} e(t)+\left(\dot{\hat{T}}(t) \hat{T}^{-1}(t)\right) e(t)+\hat{T}(t) \eta(t),
\end{aligned}
$$

$\tilde{y}(t)=C_{e} \hat{T}^{-1}(t) \tilde{x}(t)=C_{e} e(t)$,

with $e(0)=\hat{T}(0) \tilde{x}_{0}=e_{0}, \eta(t)=\tilde{A}_{e}(t) \hat{T}^{-1}(t) e(t)+E_{e} \varepsilon(t)$ and $\tau_{m}\|\tilde{x}\|^{2} \leq\|e\|^{2} \leq \tau_{M}\|\tilde{x}\|^{2}$.

Property 1 Given the error dynamic (12), the expression

$$
\delta(t)=\hat{T}(t)\left(\tilde{A}_{e}(t) x(t)(1-\sigma)+E_{e} \varepsilon(t)\right)
$$

represents part of the uncertain dynamic which cannot be compensated by the observer feedback gain $H_{t}$ in (8) for some $\sigma \in[0,1]$. If Conditions 1 4 are satisfied, the following upper bound holds:

$$
\begin{aligned}
\|\delta(t)\| & \leq \sup _{t \in \mathbb{R}^{+}}\{\|\delta(t)\|\}, \\
& \leq \tau_{M} \gamma_{E}\left(\gamma_{\tilde{\Psi}} \gamma_{\tilde{s}} \gamma_{z} \max \left\{\gamma_{z}, \gamma_{\omega}\right\}(1-\sigma)+\bar{\varepsilon}\right)=\bar{\delta},
\end{aligned}
$$

where $\gamma_{E}=\left\|E_{e}\right\|=\|E\|$.

C. Observer Design, Stability and Convergence

Lemma 1 Let $M_{1} \in \mathbb{R}^{n \times n}$ and $M_{2} \in \mathbb{R}^{n \times n}$ be arbitrary matrices for some $n \in \mathbb{N}$. If $\alpha \in \mathbb{R}^{+}$and $\beta \in \mathbb{R}^{+}$are selected so that $\gamma^{2} \leq \alpha \beta$, then the following inequality holds:

$2 \gamma\left\|M_{1} v(t)\right\|\left\|M_{2} v(t)\right\| \leq v^{T}(t)\left(\alpha M_{1}^{\top} M_{1}+\beta M_{2}^{T} M_{2}\right) v(t)$, $\forall v: \mathbb{R}^{+} \rightarrow \mathbb{R}^{n}$ bounded vector signal, $\forall t \in \mathbb{R}^{+}$.

Proof: See details in [43], [44].

Lemma 2 If the Conditions 2,3 are satisfied by (7), then the following inequality holds:

$$
\left\|e^{\top}(t) P \hat{T}(t) \tilde{A}_{e}(t) x(t) \sigma\right\| \leq \gamma\|P e(t)\|\|\bar{I} e(t)\|,
$$

in terms of its transformed states (12), where $P \in \mathbb{S}_{++}^{l+2}$, $\bar{I}=\bar{I}_{v} \bar{I}_{v}^{\top}, \bar{I}_{v}=\left[\boldsymbol{I}_{2} ; \mathbf{0}_{l \times 2}\right], \gamma=\sigma \gamma_{E} \gamma_{\theta} \gamma_{\tilde{\Psi}} \gamma_{\tilde{s}} \in \mathbb{R}^{+}$, for some $\sigma \in[0,1]$.

Proof: Considering that:

$\tilde{A}_{e}(t) x(t)=\left(A_{e}(\Psi(s(t), Q(t))-\Psi(\hat{s}(t), Q(t))) x(t)\right.$,

$=\left[\boldsymbol{I}_{2} ; \mathbf{0}_{l \times 2}\right]\left[\mathbf{0}_{2 \times 2}, E \tilde{\Psi}(t), \mathbf{0}_{2 \times l-1}\right]\left[z^{\top}(t) ; \theta(t) ; \omega_{2}(t) ; \ldots ; \omega_{l}(t)\right]$,

$=\bar{I}\left[E \tilde{\Psi} \theta(t) ; \mathbf{0}_{l \times 1}\right]$,

where $\tilde{\Psi}(t)=\Psi(s(t), Q(t))-\Psi(\hat{s}(t), Q(t))$, due to the special form $\bar{I}$, based on the Conditions $2 \sqrt[3]{3}$, with $\nu(t)=$ $\left[E \tilde{\Psi}(t) \theta(t) ; \mathbf{0}_{l \times 1}(t)\right]$ yield

$$
\begin{aligned}
e^{\top}(t) P \hat{T}(t) \tilde{A}_{e}(t) x(t) \sigma & =e^{\top}(t) W \hat{T}(t) \bar{I} \nu(t) \sigma, \\
\left\|e^{\top}(t) P \bar{I} \hat{T}(t) v(t) \sigma\right\| & \leq \sigma\left\|e^{\top}(t) P \bar{I}\right\|\|v(t)\|, \\
& \leq \sigma\left\|e^{\top}(t) P \bar{I}\right\|\|E\|\|\theta(t)\|\|\tilde{\Psi}(t)\|, \\
& \leq \underbrace{\sigma \gamma_{E} \gamma_{\theta} \gamma_{\tilde{\Psi}} \gamma_{\tilde{s}}}_{\gamma}\|P e(t)\|\|\bar{I} e(t)\|,
\end{aligned}
$$

since $\bar{I} \hat{T}(t)=\bar{I}=\bar{I} \hat{T}^{-1}(t),\|\tilde{s}(t)\| \leq \gamma_{\tilde{s}}\|\tilde{z}\|=\gamma_{\tilde{s}}\|\bar{I} e(t)\|$, for some $\sigma \in[0,1]$, where $\gamma \in \mathbb{R}^{+}$accounts for the uncertainty compensated by the observer feedback gain $H_{t}$ in $(8), \forall t \in$ $\mathbb{R}^{+}$. 
Theorem 1 Let Conditions 14 be satisfied by (7) and $e \in$ $\Omega_{r}=\left\{e(t) \in \mathbb{R}^{l+2} ;\|e(t)\|<r\right\}$ for some $r \in \mathbb{R}^{+}$. If some positive constants $\alpha$ and $\beta$ are chosen such that $\alpha \beta \geq \gamma^{2}$ and the solution for the linear matrix inequality problem:

$$
\left[\begin{array}{cc}
-\mathcal{A}^{\top}(Q(t)) P-P \mathcal{A}(Q(t))+C_{e}^{\top} Z^{\top}+Z C_{e}-\beta \bar{I}-W & \sqrt{\alpha} P \\
\sqrt{\alpha} P & \boldsymbol{I}_{l+2}
\end{array}\right] \succeq 0,
$$

exists for some $P \in \mathbb{S}_{++}^{l+2}, W \in \mathbb{S}_{++}^{l+2}, Z \in \mathbb{R}^{l+2}$ and $\rho \in(0,1]$, with $\gamma_{T} \leq c_{W} /\left(2 \rho c_{M}\right), \gamma=\sigma \gamma_{E} \gamma_{\theta} \gamma_{\tilde{\Psi}} \gamma_{\tilde{s}}, c_{m}=\lambda_{m}(P), c_{M}=$ $\lambda_{M}(P), c_{W}=\lambda_{m}(W), \forall Q \in \mathcal{Q}$, then the state observer (8) using the feedback gain

$$
H_{t}=\hat{T}^{-1}(t) P^{-1} Z
$$

guarantees that the state estimation error (11) has a EUUB dynamic [38].

Proof: Let $V=e^{\top} P e$ be a Lyapunov function candidate 5 with bounds $c_{m}\|e\|^{2} \leq V(e) \leq c_{M}\|e\|^{2}$, where $P \in$ $\mathbb{S}_{+}^{l+2}$, with $c_{m}=\lambda_{m}(P)$ and $c_{M}=\lambda_{M}(P)$. Its time derivative along the trajectory 12 yields

$$
\begin{aligned}
& \dot{V}=\dot{e}^{\top} P e+e^{\top} P \dot{e}, \\
& =e^{\top}\left(\overline{\mathcal{A}}^{\top}(t) P+P \overline{\mathcal{A}}(t)\right) e+2 e^{\top} P\left(\dot{\hat{T}} \hat{T}^{-1}\right) e+2 e^{\top} P \hat{T} \eta .
\end{aligned}
$$

If the signal $\eta$ in $[11$ is decomposed by a factor $\sigma \in[0,1]$, then $\hat{T} \eta=\delta_{0}+\delta$, where $\delta$ is given in (13) and $\delta_{0}=\hat{T} \tilde{A}_{e} x \sigma$ represents the uncertain term which can be compensated by the observer feedback term $H_{t} y(t)$ in (8). Thus, considering 16) so that $\overline{\mathcal{A}}(t)=\mathcal{A}(Q(t))-P^{-1} Z C_{e}$, 17 can be upper bounded by

$$
\begin{aligned}
& \dot{V} \leq e^{\top}\left(\mathcal{A}(Q(t))^{\top} P+P \mathcal{A}(Q(t))-C_{e}^{\top} Z^{\top}-Z C_{e}\right) e+ \\
& 2 \underbrace{\left\|e^{\top} P \hat{T} \tilde{A}_{e} x \sigma\right\|}_{T_{1}}+2\|P e\| \underbrace{\left\|\dot{\hat{T}} \hat{T}^{-1}\right\|}_{T_{2}}\|e\|+2\|P e\| \underbrace{\|\delta(t)\|}_{T_{3}} .
\end{aligned}
$$

Regarding term $T_{1}$, based on Lemma 1 and 2, the following inequality hols:

$$
2\left\|e^{\top} P \hat{T} \tilde{A}_{e} x \sigma\right\| \leq 2 \gamma\|P e\|\|\bar{I} e\| \leq e^{\top}\left(\alpha P^{\top} P+\beta \bar{I}^{\top} \bar{I}\right) e,
$$

for some $\alpha \in \mathbb{R}^{+}$and $\beta \in \mathbb{R}^{+}$so that $\gamma^{2} \leq \alpha \beta$. Similarly, with respect to term $T_{2}$ and $T_{3}$, considering Condition 4 and Property 1. respectively, an upper bound for (19) can be given by:

$$
\begin{gathered}
\dot{V} \leq e^{\top} \underbrace{\left(\mathcal{A}^{\top}(Q(t)) P+P \mathcal{A}(Q(t))-C_{e}^{\top} Z^{\top}-Z C_{e}+\alpha P P+\beta \bar{I}\right)}_{T_{4}(t)} e \\
+2 \gamma_{T} c_{M}\|e\|^{2}+2 c_{M} \bar{\delta}\|e\| .
\end{gathered}
$$

Imposing the condition $T_{4}(t) \leq-W$, for some $W \in \mathbb{S}_{+}^{l+2}$ and $\forall t \in \mathbb{R}^{+}$, by means of the Schur complement [46], [49], the resulting Riccati-like inequality [50] can be transformed to the constrained time-variant problem 15 , convex in terms of the variables $\{P, Z, W\}$. If this problem is feasible,

\footnotetext{
${ }^{5}$ For clarity, the time-dependence in most of the functions after this has been dropped.
}

decomposing $\gamma_{T}$ by a factor $\rho \in(0,1]$, and considering $e \in \Omega_{r}$, (19) can be upper bounded by:

$$
\begin{aligned}
& \dot{V} \leq-\left(c_{W}-2 \rho \gamma_{T} c_{M}\right)\|e\|^{2}+2 c_{M} \underbrace{\left(\bar{\delta}+(1-\rho) \gamma_{T} r\right)}_{\Delta}\|e\|, \quad(20) \\
& \leq-(1-\mu) \bar{c}\|e\|^{2}, \forall e \notin \Omega_{\delta}=\left\{e \in \mathbb{R}^{l+2} ;\|e\| \leq r_{\delta}=\frac{2 c_{M} \Delta}{\mu \bar{c}}\right\},
\end{aligned}
$$

with $\bar{c}=c_{W}-2 \rho \gamma_{T} c_{M}$, for some $\mu \in(0,1]$, and 20 is negative outside of the region $\Omega_{\delta}, \forall t \in \mathbb{R}^{+}$. Thus, based on the comparison lemma [48], the observer estimation error has an exponential convergence given by: $\|e(t)\| \leq$ $\sqrt{\frac{c_{M}}{c_{m}}}\left\|e_{0}\right\| \exp \left(-\frac{(1-\mu) \bar{c}}{2 c_{M}} t\right)$ towards this region (since $P, W$ and $\rho$ are such that $\left.\bar{c} \geq 0 \Rightarrow \gamma_{T} \leq c_{W} /\left(2 \rho c_{M}\right)\right)$ and this remains inside it thereafter, i.e. it is bounded [51]. In addition, considering the case of $e \in \Omega_{\delta}$, the analysis of (20) yields

$$
\begin{aligned}
& \|\tilde{x}\| \leq \sqrt{\kappa_{P}}\left(\kappa_{\hat{T}}\left\|\tilde{x}_{0}\right\| \exp \left(\frac{-\mu \bar{c}}{2 c_{M}} t\right)+\frac{r_{\delta}}{\tau_{m}} \sqrt{1-\exp \left(\frac{-\mu \bar{c}}{c_{M}} t\right)}\right), \\
& \leq \max \left\{\sqrt{\kappa_{P}} \kappa_{\hat{T}}\left\|\tilde{x}_{0}\right\|, r_{\tilde{x}}=\sqrt{\kappa_{P}} \frac{c_{M}}{\tau_{m}} \frac{2}{\mu \bar{c}} \Delta\right\},
\end{aligned}
$$

where $\kappa_{P}=c_{M} / c_{m}, \kappa_{\hat{T}}=\tau_{M} / \tau_{m}$, so that the estimation error has an exponential convergence, the ultimate bound value of which is given by $\lim _{t \rightarrow \infty}\|\tilde{x}\| \leq r_{\tilde{x}}$, i.e. this has an EUBB dynamic [38].

\section{Polytopic Design Approach}

Considering that the volumetric flow rate in (4) satisfies Condition 5, the approximate augmented model (7) admits a polytope description so that the time-variant nature of the LMI problem (15) can be recast as a polytopic convex problem [32], [52], [47], [53], [46]:

$\min _{\bar{\alpha}, \gamma_{Z}}\left\{\bar{\alpha}+\kappa_{Z} \gamma_{Z}\right\}$,

subject to:

$$
\begin{aligned}
\forall i=1,2:\{ & {\left[\begin{array}{cc}
-\mathcal{A}_{i}^{\top} P-P \mathcal{A}_{i}+C_{e}^{\top} Z^{\top}+Z C_{e}-\bar{W} & P \\
P & \bar{\alpha} \boldsymbol{I}_{l+2}
\end{array}\right] \succeq 0, } \\
& {\left[\begin{array}{cc}
\gamma_{Z} \boldsymbol{I}_{l+2} & Z \\
Z^{\top} & \gamma_{Z}
\end{array}\right] \succeq 0, } \\
& P=P^{\top} \succ 0, W=W^{\top} \succ 0, \\
& \bar{\alpha}>0, \gamma_{Z} \geq 0,
\end{aligned}
$$

with $\mathcal{A}_{1}=\mathcal{A}\left(Q_{m}\right)$ and $\mathcal{A}_{2}=\mathcal{A}\left(Q_{M}\right)$ vertices of the convex hull $\mathcal{P} ; \bar{\alpha}=1 / \alpha$ degree of freedom to maximise the uncertainty allowed by the LMI $(15)$, for a given $\beta$ and provided its feasible solution $\{P, Z, W\}$, with $\bar{W}=\beta \bar{I}+W$. The constant $\kappa_{Z} \geq 0$ is selected to adjust the norm bound of matrix $Z$.

\section{RFB ExPERIMENTAL MEthods}

The experimental setup and methodology has previously been described by the authors in [32].

Experiments were conducted in an argon-filled glovebox (PureLab, Inert Technologies, USA) using $0.1 \mathrm{M}$ vanadium acetylacetonate $\left(\mathrm{V}(\mathrm{acac})_{3}, 98 \%\right.$, Strem, UK) in anhydrous 


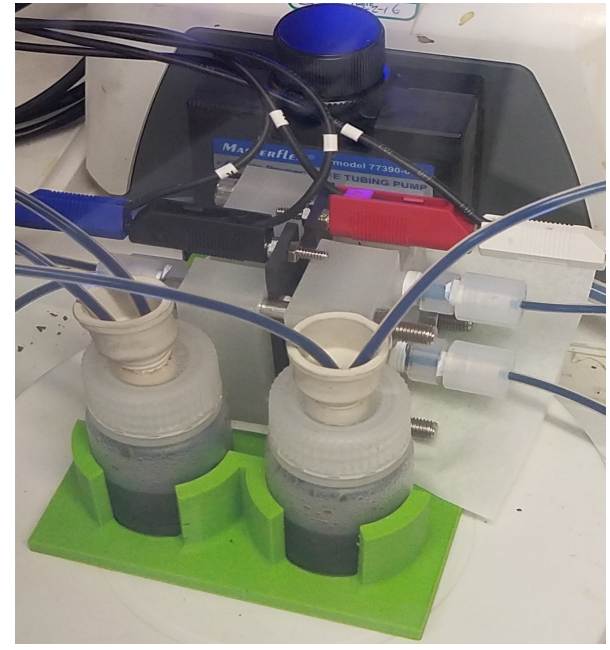

Fig. 2: RFB experimental setup

acetonitrile (ACN, 99.8\%, Sigma, UK) dried over molecular sieves ( $3 \AA$, Sigma-Aldrich, USA) with $0.2 \mathrm{M}$ tetraethylammonium tetrafluoroborate $\left(\mathrm{TEABF}_{4}, \mathrm{Sigma}, 99 \%, \mathrm{UK}\right)$ as a supporting salt.

A nonaqueous-compatible flow cell with $2.20 \mathrm{~cm}^{2}$ active area was used with reservoir volumes of $18 \mathrm{~mL}$ each and a flowrate of $9 \mathrm{~mL} / \mathrm{min}$, which corresponds to a reactor residence time of $4.65 \mathrm{~s}$ when using electrodes with a porosity $\epsilon$ of 0.87. Impervious bipolar graphite plates (GraphiteStore, USA) were used with porous carbon felt electrodes (AlfaAesar, UK) compressed $50 \%$ to a final thickness of 3.17 $\mathrm{mm}$ and used in a counter-current flow-through configuration with a porous separator (Celgard 4650, Celgard, USA).

MasterFlex peristaltic pumps (Cole-Palmer, USA) circulated electrolyte through each half-cell with polytetrafluoroethylene (PTFE) tubing using perfluoroalkoxy alkane (PFA) compression fittings. Wetted materials in the system consisted entirely of PTFE, PFA, polypropylene (PP), impervious graphite, and carbon felt. A photograph of the experimental setup is shown in Figure 2

\section{EXPERIMENTAL RESULTS}

As preconditioning procedure, the flow battery used in the experiment was cycled three times at $20 \mathrm{~mA} / \mathrm{cm}^{2}$ between $3 \mathrm{~V}$ and $1 \mathrm{~V}$. After this, the battery was charged up to the $3 \mathrm{~V}$ (voltage cutoff) to let it self-discharge at open circuit. Values of the experimental parameters in the model (4)-(5) are indicated in Table []

For comparison purposes, the following linear function for the crossover flux in (4) has been considered:

$$
Q_{x}\left(S O C_{\text {cell }}(t)\right)=k_{\mathrm{mt}} c_{0} \overbrace{\left(\frac{c_{0}-c_{\text {cell }}(t)}{c_{0}}\right)}^{S O C_{\text {cell }}(t)},
$$

where $k_{\mathrm{mt}}=5.6142 \cdot 10^{-8} \mathrm{~L} / \mathrm{min}$ is the mass-transfer coefficient. This value for $k_{m t}$ was calculated by fitting the model, (4)-(5), including (22), to the experimental data assuming an initial condition of $100 \%$ for $S O C_{\text {cell }}$ [32]. Figure 3. (a)(b) and (c) depicts the battery states and crossover term,

\begin{tabular}{c|l|l|l}
\hline Symbol & Description & Value & Units \\
\hline \hline$V_{\text {res }}$ & Reservoir volume & 17.6 & $\mathrm{~mL}$ \\
$V_{\text {cell }}$ & Half-cell volume & 0.6985 & $\mathrm{~mL}$ \\
$c_{0}$ & Initial concentration V $(\mathrm{acac}) 3$ & 0.1 & $\mathrm{~mol} / \mathrm{L}$ \\
$Q$ & Volumetric flow rate & 9.0 & $\mathrm{~mL} / \mathrm{min}$ \\
$\epsilon$ & Porosity of carbon electrode & 0.87 & - \\
$E_{\text {cell }}^{0}$ & Equilibrium cell potential & 2.2 & $\mathrm{~V}$ \\
\hline
\end{tabular}

TABLE I: Parameters values for RFB experiment

respectively (dash-dot lines) and their estimated values by the proposed observer (solid lines), alongside the estimated parameters of the model. Figure 4 shows the voltage output predicted by the model during self-discharge (dash-dot line), which is broadly in agreement with its measured value (dot line), excepting zones where $S O C_{\text {cell }}$ reaches its extreme values, caused by possibly unmodeled dynamics in the argument of the Nernst equation (5).

With respect to the proposed on-line augmented state observer $[8], \hat{z}_{0}=[0.87,0.85]^{\top}$ and $\hat{\omega}_{0}=\mathbf{0}_{l \times 1}$ have been selected as its initial conditions. For the crossover approximation (6), $s=S O C_{\text {cell }}=z_{2}, \Omega=[0,1], \Psi\left(\hat{z}_{2}(t)\right)=$ $0.5\left(1+\hat{z}_{2}(t)\right)$ and a third order pure integrator $(l=3)$ for the dynamic of the parameters, with $\lambda=[0.5,0.025]^{\top}$, have been considered. Regarding the observer gain in (16), $\varrho=10^{-4}$ has been selected to obtain a value to make the observer less sensitive to noise. The numerical solution of the polytopic LMI problem 21, considering $Q_{\mathrm{m}}=0.25 Q$, $Q_{\mathrm{M}}=2 Q, \beta=10^{-4}$ and $\kappa_{Z}=0.01$, has been obtained via the Yalmip toolbox for Matlab [54] using the SDP package part of the Mosek solver [55]. More details about its Matlab code implementation and data can be found in [56].

As it has been corroborated by the authors in [32], the states estimated by the proposed observer (see Figure 3, solid lines) describe similar behaviour to the model (4)-(5)-(22). In particular, the estimated crossover flux is in agreement with the linear relationship proposed in 22, the dynamical parametric model of which (6) helps to achieve a good tracking performance in the transient response under low magnitudes for the observer gain.

\section{CONCLUding Remarks}

In this article, for an isothermal lumped parameter model of disproportionation redox flow batteries, an observer design for the simultaneous estimation of the battery states and crossover flux have been presented. The design considers an augmented space representation of the battery model and is based on Lyapunov stability theory. The observer linear feedback gain is obtained by solving a polytopic LMI problem, providing a systematic methodology where the EUUB convergence of the augmented state estimation error is guaranteed. The crossover term has been modelledapproximated as the output of a linear differential equation, enabling continual parametric estimation of the crossover flux as the battery discharges. Data from a vanadium acetylacetonate DRFB undergoing self-discharge was analyzed, 

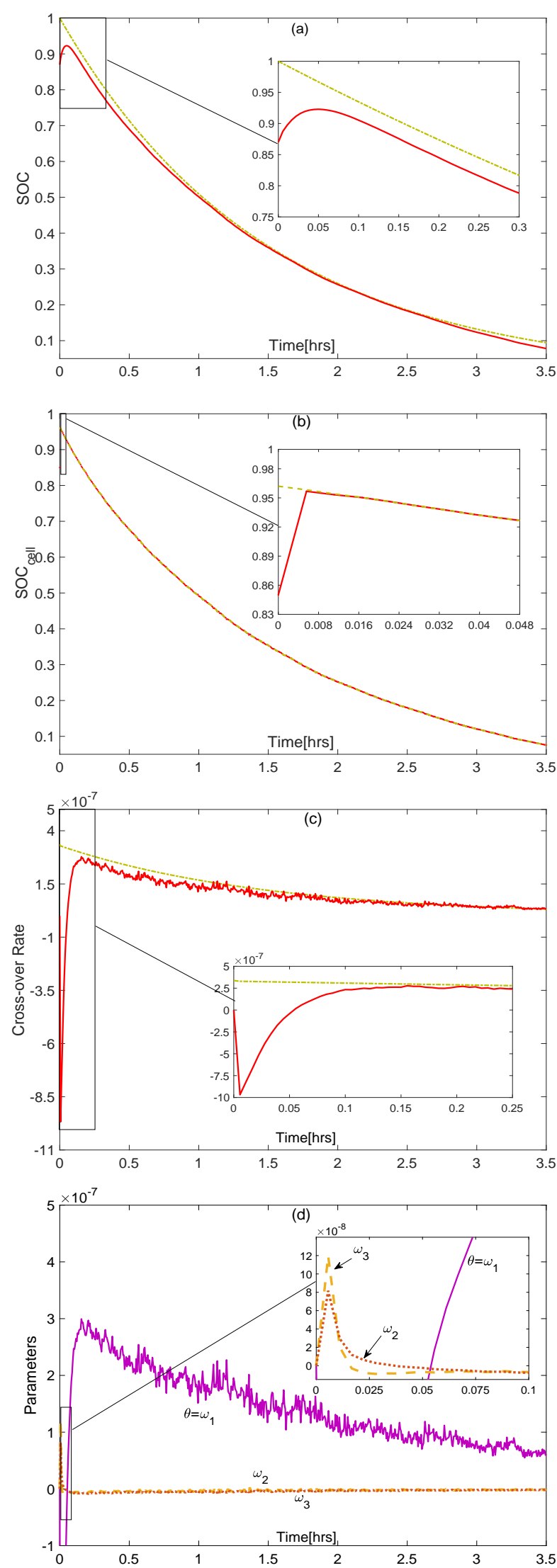

Fig. 3: Battery model (4)-(5) results with constant parameters in 22 (dash-dotted lines), alongside augmented state observer estimate (solid lines) for: (a) overall state-of-charge; (b) state-ofcharge of the half-cell; (c) crossover flux; (d) estimated parameters for 6 .

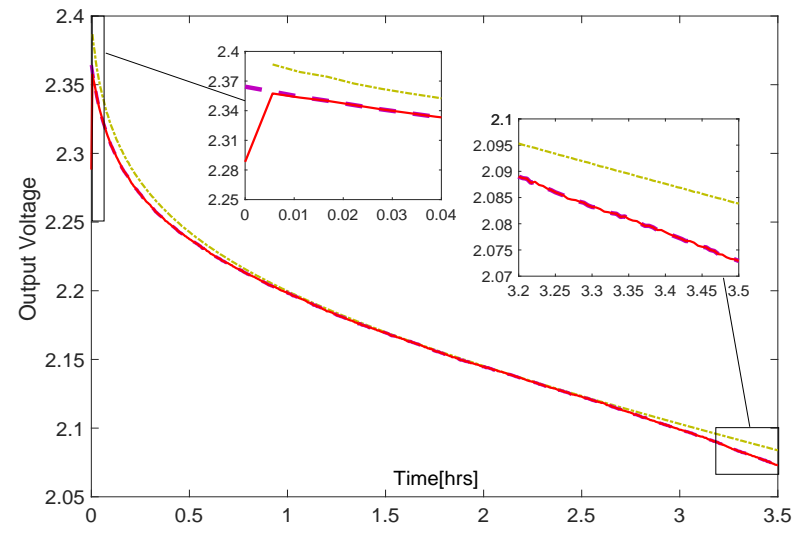

Fig. 4: Voltages with respect to self-discharge time, including: measured voltage response (dotted line); battery model (4)-(5) with constant parameters in 22 (dash-dotted line); augmented state observer estimate (solid line).

demonstrating the performance of the observer proposed, in particular with respect to transient dynamic and noise rejection in the crossover flux estimation.

\section{ACKNOWLEDGMENTS}

This work was carried out with funding support received from the Faraday Institution (faraday.ac.uk; EP/S003053/1), grant number FIRG003.

\section{REFERENCES}

[1] Adam Z. Weber, Matthew M. Mench, Jeremy P. Meyers, Philip N. Ross, Jeffrey T. Gostick, and Qinghua Liu. Redox flow batteries: a review. Journal of Applied Electrochemistry, 41(10):1137-1164, Sep 2011.

[2] Q. Xu and T.S. Zhao. Fundamental models for flow batteries. Progress in Energy and Combustion Science, 49:40-58, 2015.

[3] Q. Zheng, X. Li, Y. Cheng, G. Ning, F. Xing, and F. Zhang. Development and perspective in vanadium flow battery modeling. Applied Energy, 132:254-266, 2014.

[4] A.A. Shah, H. Al-Fetlawi, and F.C. Walsh. Dynamic modelling of hydrogen evolution effects in the all-vanadium redox flow battery. Electrochimica Acta, 55(3):1125 - 1139, 2010.

[5] Ching Liang Chen, Hak Koon Yeoh, and Mohammed Harun Chakrabarti. An enhancement to vynnycky's model for the allvanadium redox flow battery. Electrochimica Acta, 120:167 - 179, 2014.

[6] K. W. Knehr, Ertan Agar, C. R. Dennison, A. R. Kalidindi, and E. C. Kumbur. A transient vanadium flow battery model incorporating vanadium crossover and water transport through the membrane. Journal of The Electrochemical Society, 159(9):A1446-A1459, 2012.

[7] Yun Wang and Sung Chan Cho. Analysis and three-dimensional modeling of vanadium flow batteries. Journal of The Electrochemical Society, 161(9):A1200-A1212, 2014.

[8] Ao Tang, Jie Bao, and Maria Skyllas-Kazacos. Dynamic modelling of the effects of ion diffusion and side reactions on the capacity loss for vanadium redox flow battery. Journal of Power Sources, 196(24):10737-10747, 2011.

[9] A. A. Shah, R. Tangirala, R. Singh, R. G. A. Wills, and F. C. Walsh. A dynamic unit cell model for the all-vanadium flow battery. Journal of The Electrochemical Society, 158(6):A671-A677, 2011.

[10] Maria Skyllas-Kazacos and Leesean Goh. Modeling of vanadium ion diffusion across the ion exchange membrane in the vanadium redox battery. Journal of Membrane Science, 399-400:43 - 48, 2012.

[11] Philipp A. Boettcher, Ertan Agar, C. R. Dennison, and E. Caglan Kumbur. Modeling of ion crossover in vanadium redox flow batteries: A computationally-efficient lumped parameter approach for extended cycling. Journal of The Electrochemical Society, 163(1):A5244A5252, 2016. 
[12] Puiki Leung, Xiaohong Li, Carlos Ponce de León, Leonard Berlouis, C. T. John Low, and Frank C. Walsh. Progress in redox flow batteries, remaining challenges and their applications in energy storage. RSC Adv., 2:10125-10156, 2012.

[13] Massimo Guarnieri, Paolo Mattavelli, Giovanni Petrone, and Giovanni Spagnuolo. Vanadium redox flow batteries, potentials and challenges of a emerging storage technology. IEEE Industrial Electronics Magazine, 10(4):20-31, 2016.

[14] Rebecca A. Potash, James R. McKone, Sean Conte, and Héctor D. Abruña. On the benefits of a symmetric redox flow battery. Journal of The Electrochemical Society, 163(3):A338-A344, 2016.

[15] Xianfeng Li, Huamin Zhang, Zhensheng Mai, Hongzhang Zhang, and Ivo Vankelecom. Ion exchange membranes for vanadium redox flow battery (vrb) applications. Energy Environ. Sci., 4:1147-1160, 2011.

[16] Seongyeon Won, Kyeongmin Oh, and Hyunchul Ju. Numerical analysis of vanadium crossover effects in all-vanadium redox flow batteries. Electrochimica Acta, 177:310 - 320, 2015. International Conference on Electrochemical Energy Science and Technology (EEST2014).

[17] D. Schmal, J. Van Erkel, and P. J. Van Duin. Mass transfer at carbon fibre electrodes. Journal of Applied Electrochemistry, 16(3):422-430, May 1986.

[18] E. Wiedemann, A. Heintz, and R.N. Lichtenthaler. Transport properties of vanadium ions in cation exchange membranes:: Determination of diffusion coefficients using a dialysis cell. Journal of Membrane Science, 141(2):215 - 221, 1998

[19] Chenxi Sun, Jian Chen, Huamin Zhang, Xi Han, and Qingtao Luo. Investigations on transfer of water and vanadium ions across nafion membrane in an operating vanadium redox flow battery. Journal of Power Sources, 195(3):890-897, 2010.

[20] Jovan Kamcev, Donald R. Paul, Gerald S. Manning, and Benny D. Freeman. Accounting for frame of reference and thermodynamic nonidealities when calculating salt diffusion coefficients in ion exchange membranes. Journal of Membrane Science, 537:396-406, 2017.

[21] Tao Luo, Said Abdu, and Matthias Wessling. Selectivity of ion exchange membranes: A review. Journal of Membrane Science, 555:429 - 454, 2018.

[22] Aaron A. Shinkle, Alice E.S. Sleightholme, Lucas D. Griffith, Levi T. Thompson, and Charles W. Monroe. Degradation mechanisms in the non-aqueous vanadium acetylacetonate redox flow battery. Journal of Power Sources, 206:490 - 496, 2012.

[23] Y. Ashraf Gandomi, D. S. Aaron, J. R. Houser, M. C. Daugherty, J. T. Clement, A. M. Pezeshki, T. Y. Ertugrul, D. P. Moseley, and M. M. Mench. Critical review-experimental diagnostics and material characterization techniques used on redox flow batteries. Journal of The Electrochemical Society, 165(5):A970-A1010, 2018.

[24] M.R. Mohamed, H. Ahmad, M.N. Abu Seman, S. Razali, and M.S. Najib. Electrical circuit model of a vanadium redox flow battery using extended kalman filter. Journal of Power Sources, 239:284 - 293, 2013.

[25] Binyu Xiong, Jiyun Zhao, Zhongbao Wei, and Maria Skyllas-Kazacos. Extended kalman filter method for state of charge estimation of vanadium redox flow battery using thermal-dependent electrical model. Journal of Power Sources, 262:50 - 61, 2014.

[26] Zhongbao Wei, Tuti Mariana Lim, Maria Skyllas-Kazacos, Nyunt Wai, and King Jet Tseng. Online state of charge and model parameter coestimation based on a novel multi-timescale estimator for vanadium redox flow battery. Applied Energy, 172:169 - 179, 2016.

[27] B. Xiong, J. Zhao, Y. Su, Z. Wei, and M. Skyllas-Kazacos. State of charge estimation of vanadium redox flow battery based on sliding mode observer and dynamic model including capacity fading factor. IEEE Transactions on Sustainable Energy, 8(4):1658-1667, Oct 2017.

[28] Zhongbao Wei, Arjun Bhattarai, Changfu Zou, Shujuan Meng, Tuti Mariana Lim, and Maria Skyllas-Kazacos. Real-time monitoring of capacity loss for vanadium redox flow battery. Journal of Power Sources, 390:261 - 269, 2018.

[29] Chen D. A Yu V, Headley A. Constrained extended kalman filter for state-of-charge estimation of a vanadium redox flow battery with crossover effects. ASME. J. Dyn. Sys., Meas., Control, 136(4):041013041013-7, 2014

[30] Qinghua Liu, Alice E.S. Sleightholme, Aaron A. Shinkle, Yongdan $\mathrm{Li}$, and Levi T. Thompson. Non-aqueous vanadium acetylacetonate electrolyte for redox flow batteries. Electrochemistry Communications, 11(12):2312-2315, 2009.

[31] James D. Saraidaridis and Charles W. Monroe. Nonaqueous vanadium disproportionation flow batteries with porous separators cycle stably and tolerate high current density. Journal of Power Sources, 412:384 $-390,2019$.

[32] P. Ascencio, K. Smith, C. W. Monroe, and D. Howey. Adaptive observer for charge-state and crossover estimation in disproportionation redox flow batteries undergoing self-discharge. Accepted in ACC: American Control Conference, July 10-12, 2019.

[33] James D. Saraidaridis. Analysis and performance of symmetric nonaqueous redox flow batteries. $\mathrm{PhD}$ thesis, University of Oxford, Department of Engineering Science, 2017.

[34] C.D. Johnson. Accommodation of external disturbaces in linear regulator and servomechanism problems. IEEE Transactions on Automatic Control, 16(6):635-644, 1971.

[35] D. Sbarbaro, P. Ascencio, and S. Feyo de Azevedo. An enhanced model for parameter estimation in bio-processes. In UKACC International Conference on Control, University of Cambridge, 2000.

[36] P. Ascencio and D. Sbarbaro. An ekf based on enhanced model for parameter estimation in bio-processes. In ECC, European Contro Conference, University of Porto, 2001.

[37] H. K. Khalil. Nonlinear Systems. Pearson, 3rd edition, 2002.

[38] M. Farza, H. Hammouri, S. Othman, and K. Busawon. Nonlinear observers for parameter estimation in bioprocesses. Chemical Engineering Science, 52(23):4251-4267, 1997.

[39] M. Farza, K. Busawon, and H. Hammouri. Observers for on-line estimation of kinetic rates in bioreactors. Automatica, 34(3):301-318, 1998.

[40] K. Busawon, M. Farza, and H. Hammouri. Observers design for a special class of non-linear systems. International Journal of Control, 71(3):405-418, 1998.

[41] M. Nadri, M. Farza, H. Hammouri, and M. Fick. Nonlinear observers for state and parameter estimation in biochemical processes. In Proceeding of the IFAC, Barcelona, Spain, 2002.

[42] S. Raghavan and J.K. Hedrick. Observer design for a class of nonlinear systems. Int. Journal of Control, 59(2):515-528, 1994.

[43] K. Reif, F. Sonnemann, and R. Unbehauen. Nonliner state observation using $h_{\infty}$-filtering riccati desing. IEEE Transactions on Automatic Control, 44(1):203-208, 1999.

[44] P. Ascencio, D. Sbarbaro, and S. Feyo de Azevedo. An adaptive fuzzy hybrid state observer for bioprocesses. IEEE Transactions on Fuzzy Systems, 12(5):641-651, 2004.

[45] Gildas Besançon (Ed.). Nonlinear Observers and Applications. Springer-Verlag, 2007.

[46] Stephen Boyd, Laurent El Ghaoui, Eric Feron, and Venkataramanan Balakrishnan. Linear Matrix Inequalities in System and Control Theory. SIAM, 1994

[47] Floriane Anstett, Gilles Millérioux, and Gérard Bloch. Polytopic observer design for lpv systems based on minimal convex polytope finding. Journal of Algorithms \& Computational Technology, 3(1):2343, 2009

[48] H.K. Khalil. Nonlinear Systems. Prentice Hall, Upper Saddle River, N.Y., 1996.

[49] Young Man Cho and R. Rajamani. A systematic approach to adaptive observer synthesis for nonlinear systems. IEEE Transactions on Automatic Control, 42(4):534-537, Apr 1997.

[50] K. Reif, F. Sonnemann, and R. Unbehauen. Nonlinear state observation using $\mathrm{h} / \mathrm{sub} / \mathrm{spl}$ infin//-filtering riccati design. IEEE Transactions on Automatic Control, 44(1):203-208, Jan 1999.

[51] A. Jafari and A. Zinober. Sliding mode controller-observer systems with unmatched uncertainty. In Proceeding of the IFAC, Beijing, P.R. China, 1999.

[52] P. Ascencio, D. Sbarbaro, and S. F. de Azevedo. An adaptive fuzzy hybrid state observer for bioprocesses. IEEE Transactions on Fuzzy Systems, 12(5):641-651, Oct 2004.

[53] G. Millerioux, L. Rosier, G. Bloch, and J. Daafouz. Bounded state reconstruction error for $1 \mathrm{pv}$ systems with estimated parameters. IEEE Transactions on Automatic Control, 49(8):1385-1389, Aug 2004.

[54] J. Löfberg. Yalmip : A toolbox for modeling and optimization in matlab. [Online]. Available: http://users.isy.liu.se/johanl/yalmip, 2004

[55] The mosek optimization toolbox for matlab, version 8. Denmark: MOSEK ApS. [Online]. Available: https://www.mosek.com/.

[56] Matlab code: Augmented state observer for simultaneous estimation of charge state and crossover in self-discharging disproportionation redox flow batteries. [Online] Available: https://github.com/davidhowey/CCTA2019, 2019. 